\title{
Preparation and Characterization of Protein-loaded Lipid-polymer Hybrid Nanoparticles with Polycaprolactone as Polymeric Core Material
}

\section{Burcu Devrim* and Asuman Bozkır}

Ankara University, Faculty of Pharmacy, Department of Pharmaceutical Technology, Tandogan, Ankara, Turkey

\begin{abstract}
Lipid-polymer hybrid nanoparticles (LPNs) have emerged as a potent therapeutic nano-carrier alternative to liposomes and polymeric nanoparticles. In this work, lipid-polymer hybrid nanoparticles were prepared using polycaprolactone, phosphatidylcholine: glyceryl tripalmitate mixture and lysozyme as the polymer, lipids and model protein, respectively. Uniform nanoparticles with about $100 \mathrm{~nm}$ in size were obtained using the modified emulsification solvent evaporation method. The results indicated that LPNs showed higher encapsulation efficiency compared with naked polycaprolactone nanoparticles. According to the results of bioactivity assay, $63.86 \%$ bioactive lysozyme was recovered from the LPNs. These results indicated that modification of polycaprolactone nanoparticles with lipids could considerably increase the drug-delivery efficiency and LPNs had potential in the delivery of peptides and proteins.
\end{abstract}

Keywords: Nanomedicine; Lipid-polymer hybrid nanoparticles; Polycaprolactone; Lipids; Protein delivery; Lysozyme

\section{Introduction}

Use of nanoparticle has shown great potential as novel drug delivery systems. The ability to deliver drugs more effectively and efficiently to the site of interest translates into less harmful systemic side effects and more beneficial therapeutic action [1]. Nanoparticulate delivery systems such as liposomes and polymeric nanoparticles (NPs) have been extensively developed for delivering a variety of bioactive molecules such as drugs, genes, proteins, and targeting ligands $[2,3]$. Among these delivery systems, liposomes are spherical lipid vesicles with a bilayer structure of natural or synthetic amphiphilic lipid molecules. Liposomes have been widely used as drug delivery vesicles because of their biocompatible, biodegradable, nontoxic, flexible, and non-immunogenic properties for systemic and local administration. Furthermore, the lipid materials are able to fuse with the cell membrane and enhance the cell internalization of liposomes. However, liposomes have several limitations from the viewpoint of physical and chemical stability, batch-to-batch reproducibility, sterilization and manufacturing scale-up [4,5]. On the other hand, polymeric nanoparticles possess high structural integrity afforded by the rigidity of the polymer matrix, and are thus inherently more stable than liposomes [6,7]. The limitations of polymeric NPs include use of toxic organic solvents in the production process, poor drug encapsulation for hydrophilic drugs, polymer cytotoxicity and polymer degradation [8].

The lipid-polymer hybrid NPs (LPNs) combining the positive attributes of both liposomes and polymeric NPs are increasingly being considered as promising candidates to carry therapeutic agents safely and efficiently $[9,10]$. LPNs are polymeric nanoparticles enveloped by lipid layers [11]. The inclusion of lipid coat enables the hybrid nanoparticles to encapsulate not only water-insoluble drugs, but also water-soluble drugs with greater encapsulation efficiency. LPNs exhibit high structural integrity, stability during storage, and controlled release capability attributed to the polymer core, and high biocompatibility and bioavailability owed to the lipid layer $[12,13]$.

Poly- $\varepsilon$-caprolactone (PCL) is a widely used Food and Drug Administration (FDA) approved polymer which has been used in the preparation of NPs. PCL is a biocompatible and biodegradable polymer which is non-toxic and has great permeability to several drugs. Degradation of PCL does not result in an acidic environment which might disrupt the structure and properties of proteins, unlike the other commonly used biodegradable polymers such as poly(lactide) (PLA) and poly (lactide-co-glycolide) (PLGA) [14].

Among some of the technologies developed to prepare LPNs, the polymeric core and lipid shell are prepared separately using two independent processes; then the two components are combined by direct hydration, sonication, or extrusion to obtain the desired lipid shell polymer core structure in a two-step procedure. However, some limitations of this method are the technical complexity and less efficient processes of preparing both polymeric core and liposomes vesicles separately. To circumvent these problems, a relatively simple approach that combines the dual steps of the two-step method into a single step has been evaluated. Variations of the single-step method have been reported in the literature, including modified solvent extraction/ evaporation and nanoprecipitation methods [15].

In this paper, we successfully devised and characterized LPNs for the delivery of proteins/peptides which can preserve their stability and release them in a controlled way. Lysozyme was used as a model protein. PCL was chosen as the polymeric core material due to its great biodegradability and biocompatability; phosphatidylcholine (PC) and glyceryl tripalmitate were selected as lipids. The modified water1-inoil-in-water2 (w1/o/w2) emulsion method was used to prepare the LPNs. The resulting LPNs were characterized for their physicochemical properties. Additionally, the enzyme activity of lysozyme was determined by using turbidimetric method.

${ }^{*}$ Corresponding author: Burcu Devrim, Ankara University, Faculty of Pharmacy, Department of Pharmaceutical Technology, 06100 Tandoğan, Ankara, Turkey, Tel: +90 312 2033162; Fax: +90 312 2131081; E-mail: bdevrim@pharmacy.ankara.edu.tr

Received May 19, 2014; Accepted June 28, 2014; Published June 30, 2014

Citation: Devrim B, Bozkır A (2014) Preparation and Characterization of Proteinloaded Lipid-polymer Hybrid Nanoparticles with Polycaprolactone as Polymeric Core Material. J Biomol Res Ther 3: 115. doi: 10.4172/2167-7956.1000115

Copyright: (C) Devrim B, et al. This is an open-access article distributed under the terms of the Creative Commons Attribution License, which permits unrestricted use, distribution, and reproduction in any medium, provided the original author and source are credited. 
Citation: Devrim B, Bozkır A (2014) Preparation and Characterization of Protein-loaded Lipid-polymer Hybrid Nanoparticles with Polycaprolactone as Polymeric Core Material. J Biomol Res Ther 3: 115. doi: 10.4172/2167-7956.1000115

\section{Experimental}

\section{Materials}

Lysozyme, egg white was obtained from Vivantis (USA). Poly( $\varepsilon$ caprolactone) (PCL) (Mw=14 kDa) was purchased from Aldrich (USA). L-a-phosphatidylcholine (PC), glyceryl tripalmitate and Pluronic F-127 were purchased from Sigma (USA). Dichloromethane (DCM) $(99.9 \%$, HPLC grade) and Micrococcus lysodeikticus (ATCC No. 4698) were obtained from Sigma-Aldrich (Germany). All other chemicals used were analytical grade.

\section{Preparation of lipid-polymer hybrid nanoparticle (LPNs)}

The LPNs were prepared by a modified w1/o/w2 doubleemulsification-solvent-evaporation method. Briefly, 100, 200 or $300 \mathrm{mg}$ PCL and $45 \mathrm{mg}$ PC:glyceryl tripalmitate mixture were dissolved in $8 \mathrm{ml}$ DCM:acetone mixture as organic solvents to form the oil phase, while $10 \mathrm{mg}$ of lysozyme was dissolved in $1 \mathrm{ml}$ aqueous Tween 80 or sodium dodecyl sulfate (SDS) solution to form the internal aqueous phase (w1). Next, the aqueous protein solution was emulsified in the organic solution by sonication for $2 \mathrm{~min}$ in an ice bath. The resultant nanoemulsion was poured into $10 \mathrm{ml}$ 1\% (w/v) Pluronic F-127 solution (w2) and was sonicated again for $5 \mathrm{~min}$ in an ice bath. Afterwards, the nanoemulsion was stirred overnight at room temperature to evaporate off organic solvents, and the resultant NPs were collected by centrifugation using Vivaspin 20 centrifugal concentrator (Sigma-Aldrich, USA), washed with ultrapure water (MilliQ water) and lyophilized to obtain free flowing powder. Compositions used for LPNs are shown in Table 1.

\section{Physicochemical characterizations of lysozyme-loaded LPNs}

The size, polydispersity index and zeta potential of the LPNs in an aqueous suspension were measured by dynamic light scattering (DLS) using the Malvern Zetasizer (Malvern, UK); all measurements were taken at $25^{\circ} \mathrm{C}$.

The drug encapsulation efficiency was determined from the ratio of the encapsulated lysozyme to the lysozyme initially added. The amount of lysozyme was determined by using microBCA protein assay reagent kit according to the instructions of the manufacturer $[6,16]$. All experiments were performed in triplicate. Background readings were corrected for using the supernatant of blank LPNs of a corresponding batch. The protein loading was determined from the ratio of the encapsulated protein amount to the whole LPNs mass (i.e. protein+polymer+lipid) [17]. The LPNs production yield was determined from Equation (1). Production yield $(\%)=($ Total LPNs amount (mg))/(Total solid material amount (mg)) x100 (1).

\section{Estimation of bioactivity of lysozyme}

Lysozyme activity was determined using the decrease in optical

\begin{tabular}{|c|c|c|c|c|}
\hline $\begin{array}{c}\text { Formulation } \\
\text { code }\end{array}$ & $\begin{array}{c}\text { Internal } \\
\text { aqueous phase }\end{array}$ & $\begin{array}{c}\text { Amount of } \\
\mathbf{P C L}(\mathbf{m g})\end{array}$ & $\begin{array}{c}\text { Amount of } \\
\mathbf{P C}(\mathbf{m g})\end{array}$ & $\begin{array}{c}\text { Amount of glyceryl } \\
\text { tripalmitate (mg) }\end{array}$ \\
\hline NPN1 & $5 \%$ SDS & 200 & - & - \\
\hline LPN1 & $5 \%$ Tween 80 & 200 & 9 & 36 \\
\hline LPN2 & $5 \%$ SDS & 100 & 9 & 36 \\
\hline LPN3 & $5 \%$ SDS & 200 & 9 & 36 \\
\hline LPN4 & $5 \%$ SDS & 300 & 9 & 36 \\
\hline LPN5 & $5 \%$ SDS & 200 & 15 & 30 \\
\hline LPN6 & $5 \%$ SDS & 200 & 22.5 & 22.5 \\
\hline LPN7 & $5 \%$ SDS & 200 & 30 & 15 \\
\hline LPN8 & $5 \%$ SDS & 200 & 36 & 9 \\
\hline
\end{tabular}

dispersion at $450 \mathrm{~nm}$ of a M. lysodeikticus suspension. Briefly, $0.2 \mathrm{mg} /$ $\mathrm{ml}$ dispersion of M. lysodeikticus (Sigma-Aldrich, USA) was prepared in a $66 \mathrm{mM}$ phosphate buffer $\mathrm{pH}$ 6.6. To $2.9 \mathrm{ml}$ of this suspension, $0.1 \mathrm{ml}$ of the lysozyme-containing solution from the encapsulation efficiency was added, and the decrease in time of the OD450 $\mathrm{nm}$ monitored. Enzyme activity was deduced from the slope of the curve [18].

\section{Statistical analysis}

Results were expressed as mean \pm standard deviation (SD) from at least three separate measurements. A one-way analysis of variance followed by post hoc Tukey's multiple comparison tests was used to assess statistical difference. All analyses were performed by SPSS for Windows statistical software version 11.5. Significance was established when the $p$ value was less than 0.05 .

\section{Results and Discussion}

Herein we investigate the feasibility of creating LPNs containing PCL and PC:glyceryl tripalmitate mixture as the polymer and lipid constituents, respectively. The PCL is one of the most widely used biodegradable polyester in the preparation of nanoparticles due to its great biocompatibility and biodegradability properties [14]. However, it is not water soluble and this poses a significant challenge to encapsulating hydrophilic drugs into water-insoluble polymers efficiently. The hydrophobic ion-pairing (HIP) technique has attracted great interest in the field of water-soluble protein/peptide delivery [19]. With the HIP technique, it is possible to increase the liposolubility of the protein by the complex formed, thereby increasing the solubility of more protein/peptides in organic solvents, and allowing homogenous mixing of the complex with water-insoluble polymers materials for encapsulating more protein/peptides [20]. Furthermore, for many proteins, this solution in organic solvents occurs with retention of their native structure and maintenance of their enzymatic activity [21]. In this study, lysozyme was combined with sodium dodecyl sulfate (SDS) to increase its lipophilicity. While $20.80 \%$ of encapsulation efficiency was obtained with $5 \%(\mathrm{w} / \mathrm{v})$ Tween 80 as emulsifier in the inner aqueous phase, use of $5 \%(\mathrm{w} / \mathrm{v})$ SDS solution as the inner aqueous phase caused a significant increase in the encapsulation efficiency of LPNs. Additionally, a significant decrease occurred in the size of the LPNs by using SDS as emulsifier for primary emulsification (Table 2). This decrease in particle size is believed to be due to more stable primary emulsion depending on using lysozyme: SDS complex in the inner aqueous phase. The stabilization effect led to the decrease in the size of LPNs.

Effects of PCL concentration on the size of LPNs were found to be similar for non-hybrid polymeric NPs. It was observed that, as the amount of PCL was increased from $100 \mathrm{mg}$ to $300 \mathrm{mg}$, the size of LPNs increased significantly $(\mathrm{p}<0.05)$. The increase in LPNs size could

\begin{tabular}{|c|c|c|c|c|}
\hline $\begin{array}{c}\text { Formulation } \\
\text { code }\end{array}$ & $\begin{array}{c}\text { Particle size } \\
\mathbf{( n m})\end{array}$ & $\begin{array}{c}\text { Polydispersity } \\
\text { index }\end{array}$ & $\begin{array}{c}\text { Zeta Potential } \\
\mathbf{( m V )}\end{array}$ & Yield (\%) \\
\hline NPN1 & $107.9 \pm 0.40$ & $0.18 \pm 0.001$ & $-3.28 \pm 0.19$ & 68.60 \\
\hline LPN1 & $470.4 \pm 22.80$ & $0.66 \pm 0.048$ & $-8.30 \pm 0.34$ & 91.36 \\
\hline LPN2 & $90.2 \pm 0.24$ & $0.100 \pm 0.013$ & $-6.52 \pm 0.082$ & 85.00 \\
\hline LPN3 & $116.57 \pm 1.84$ & $0.45 \pm 0.007$ & $-5.82 \pm 0.31$ & 85.00 \\
\hline LPN4 & $121.7 \pm 2.86$ & $0.36 \pm 0.014$ & $-8.90 \pm 0.18$ & 73.63 \\
\hline LPN5 & $112.32 \pm 0.30$ & $0.095 \pm 0.009$ & $-10.9 \pm 0.40$ & 71.82 \\
\hline LPN6 & $117.1 \pm 2.63$ & $0.33 \pm 0.031$ & $-10.5 \pm 0.058$ & 64.09 \\
\hline LPN7 & $110.43 \pm 1.68$ & $0.29 \pm 0.024$ & $-21.77 \pm 0.93$ & 55.25 \\
\hline LPN8 & $124.53 \pm 2.26$ & $0.29 \pm 0.004$ & $-17.33 \pm 1.27$ & 57.46 \\
\hline
\end{tabular}

Table 2: Physicochemical characterization of lipid-polymer hybrid NPs. 
Citation: Devrim B, Bozkır A (2014) Preparation and Characterization of Protein-loaded Lipid-polymer Hybrid Nanoparticles with Polycaprolactone as Polymeric Core Material. J Biomol Res Ther 3: 115. doi: 10.4172/2167-7956.1000115

be explained on the basis of the viscosity of organic phase which was increased due to higher concentration of the PCL and caused larger droplets in the emulsion resulting in increased size. Similarly, the improved entrapment efficiency with higher amount of PCL could be explained on the basis of PCL viscosity in the organic phase (Figure 1a).

The PC:glyceryl tripalmitate lipid layer functions as a surfactant stabilizer of the w1/o/w2 emulsion, and subsequently functions as stabilizer of the NP suspension upon evaporation of the oil phase. Therefore, the optimal amount of lipid required to prepare the LPNs were determined. As shown in Table 2, the size of the naked PCL NPs (NPN1) was not different significantly from LPNs $(\mathrm{p}>0.05)$.

The results indicated that the size of LPNs were not significantly changed with increasing the ratio of PC:glyceryl tripalmitate $(p>0.05)$. On the other hand, the PC:glyceryl tripalmitate ratio was found to influence encapsulation efficiency of LPNs. As shown in Figure 1b, higher PC:glyceryl tripalmitate ratios led to higher encapsulation efficiencies. Lipid layer acts as a molecular barrier that keeps the encapsulated substance inside the polymer during the encapsulation process, resulting in high encapsulation efficiency [20]

a)

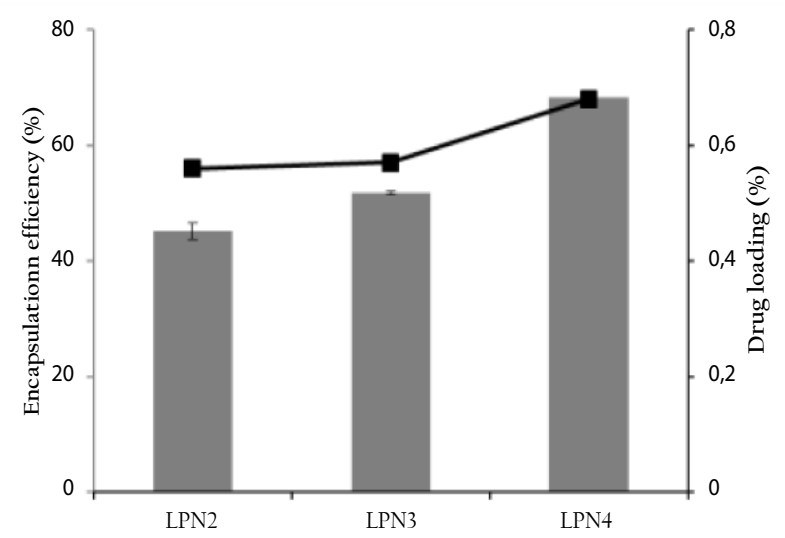

b)

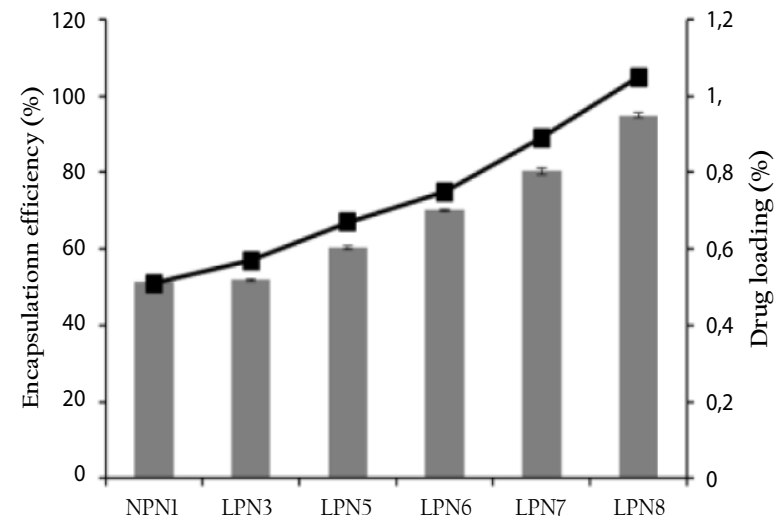

Figure 1: Encapsulation efficiencies and drug loading values of lipid-polymer hybrid NPs.

When concentration of PCL in organic phase was increased from $100 \mathrm{mg}$ to $300 \mathrm{mg}$, the zeta potentials of LPNs were not significantly changed $(p>0.05)$. However, the zeta potentials of LPNs were tended to decrease (more negative) with increasing the ratio of PC:glyceryl tripalmitate.

The production yield was defined as the mass of LPNs produced divided by the initial mass of the polymer and the lipid. According to Table 2, the production yield of range $55.25-91.36 \%$ in the LPNs formulations was obtained.

One of the major problems associated with solvent evaporation method is instability arising during particles formulation. The primary emulsion is produced by dispersing an aqueous solution of a protein into an immiscible organic solution of polymer. During this process, the protein is exposed at the interface to the organic solvent resulting its denaturation. Denaturation of proteins during lyophilization and during storage also decreases the potency of entrapped protein [21]. Thus, to determine effect of encapsulation process on the biological activity of lysozyme, bioactivity assay was carried out on the lysozyme extracted from LPNs by using turbidimetric method. According to the results of bioactivity assay, $63.86 \%$ bioactive lysozyme was recovered from the LPNs (Figure 2).

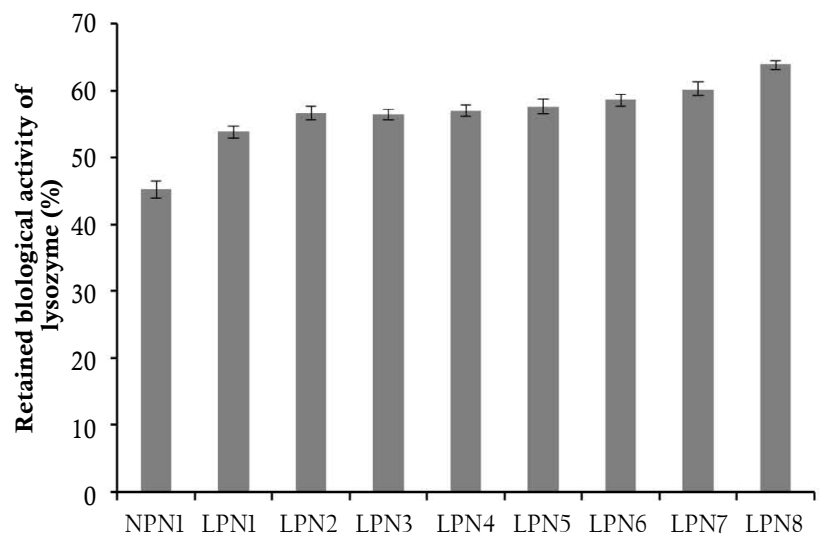

Figure 2: Retained lysozyme bioactivity of lipid-polymer hybrid NPs.

\section{Conclusions}

The lysozyme-loaded LPNs were successfully formulated by using PCL as hydrophobic polymeric core material. Physicochemical properties of LPNs were depended from concentration of PCL and ratio of PC:glyceryl tripalmitate. Additionally, preparation of LPNs succeeded to maintain the biological activity of the encapsulated proteins. Therefore, we believe that the LPNs prepared by using PCL are an attractive alternative to prepare peptide/protein delivery systems.

\section{References}

1. Clawson C, Ton L, Aryal S, Fu V, Esener S, et al. (2011) Synthesis and characterization of lipid-polymer hybrid nanoparticles with $\mathrm{pH}$-triggered poly(ethylene glycol) shedding. Langmuir 27: 10556-10561.

2. Olubummo A, Schulz M, Lechner BD, Scholtysek P, Bacia K, et al. (2012) Controlling the localization of polymer-functionalized nanoparticles in mixed lipid/polymer membranes. ACS Nano 6: 8713-8727.

3. Desai PR, Marepally S, Patel AR, Voshavar C, Chaudhuri A, et al. (2013) Topical delivery of anti-TNFÎ \pm siRNA and capsaicin via novel lipid-polymer hybrid nanoparticles efficiently inhibits skin inflammation in vivo. J Control Release 170: 51-63.

4. Sharma A, Sharma US (1997) Liposomes in drug delivery: progress and limitations. Int J Pharm 154:123-140.

5. Mandal B, Bhattacharjee H, Mittal N, Sah H, Balabathula P, et al. (2013) Core-shell-type lipid-polymer hybrid nanoparticles as a drug delivery platform. Nanomedicine 9: 474-491.

6. Cheow WS, Hadinoto K (2011) Factors affecting drug encapsulation and stability of lipid-polymer hybrid nanoparticles. Colloids Surf B Biointerfaces 85 214-220. 
Citation: Devrim B, Bozkır A (2014) Preparation and Characterization of Protein-loaded Lipid-polymer Hybrid Nanoparticles with Polycaprolactone as Polymeric Core Material. J Biomol Res Ther 3: 115. doi: 10.4172/2167-7956.1000115

7. Pinto-Alphandary $\mathrm{H}$, Andremont A, Couvreur $\mathrm{P}$ (2000) Targeted delivery of antibiotics using liposomes and nanoparticles: research and applications. Int $\mathrm{J}$ Antimicrob Agents 13: 155-168

8. Pinto Reis C, Neufeld RJ, Ribeiro AJ, Veiga F (2006) Nanoencapsulation I. Methods for preparation of drug-loaded polymeric nanoparticles. Nanomedicine 2: 8-21.

9. Yang Z, Luo X, Zhang X, Liu J, Jiang Q (2013) Targeted delivery of 10-hydroxycamptothecin to human breast cancers by cyclic RGD-modified lipid-polymer hybrid nanoparticles. Biomed Mater 8: 025012.

10. Fang RH, Aryal S, Hu CM, Zhang L (2010) Quick synthesis of lipid-polyme hybrid nanoparticles with low polydispersity using a single-step sonication method. Langmuir 26: 16958-16962.

11. Wang Y, Kho K, Cheow WS, Hadinoto K (2012) A comparison between spray drying and spray freeze drying for dry powder inhaler formulation of drugloaded lipid-polymer hybrid nanoparticles. Int J Pharm 424: 98-106.

12. Chan JM, Zhang L, Yuet KP, Liao G, Rhee JW, et al. (2009) PLGA-lecithin$P E G$ core-shell nanoparticles for controlled drug delivery. Biomaterials 30: $1627-1634$

13. Hadinoto K, Sundaresan A, Cheow WS (2013) Lipid-polymer hybrid nanoparticles as a new generation therapeutic delivery platform: a review. Eur J Pharm Biopharm 85: 427-443.

14. Rayaprolu BM, Strom JG (2013) Design and evaluation of D-atocophery polyethylene glycol 1000 succinate emulsified poly-Ï $\mu$-caprolactone nanoparticles for protein/peptide drug delivery. Drug Devlnd Pharm 39: 1046-1052.

15. Devrim B, Bozkir A, Canefe K (2011) Preparation and evaluation of PLGA microparticles as carrier for the pulmonary delivery of rhIL-2: I. Effects of some formulation parameters on microparticle characteristics. J Microencapsul 28 : 582-594.

16. Devrim B, Bozkır A (2013) Preparation and evaluation of double-walled microparticles prepared with a modified water-in-oil-in-oil-in-water (w1/o/o/w3) method. J Microencapsul 30: 741-754.

17. Meyer JD, Manning MC (1998) Hydrophobic ion pairing: altering the solubility properties of biomolecules. Pharm Res 15: 188-193.

18. Dai WG, Dong LC (2007) Characterization of physiochemical and biological properties of an insulin/lauryl sulfate complex formed by hydrophobic ion pairing. Int J Pharm 336: 58-66.

19. Yang L, Cui F, Shi K, Cun D, Wang R (2009) Design of high payload PLGA nanoparticles containing melittin/sodium dodecyl sulfate complex by the hydrophobic ion-pairing technique. Drug DevInd Pharm 35: 959-968.

20. Su X, Wang Z, Li L, Zheng M, Zheng C, et al. (2013) Lipid-polymer nanoparticles encapsulating doxorubicin and 2'-deoxy-5-azacytidine enhance the sensitivity of cancer cells to chemical therapeutics. Mol Pharm 10: 1901-1909.

21. Srinivasan C, Katare YK, Muthukumaran T, Panda AK (2005) Effect of additives on encapsulation efficiency, stability and bioactivity of entrapped lysozyme from biodegradable polymer particles. J Microencapsul 22: 127-138. 